\title{
Evaluating the terminal-velocity assumption in simulations of long-range inert ember transport
}

\author{
C.M. Thomas $^{a b}$, J.J. Sharples ${ }^{a b}$ and J.P. Evans ${ }^{c}$ \\ aAplied and Industrial Mathematics Research Group, School of Physical, Environmental and Mathematical \\ Sciences, UNSW Canberra, Australia \\ ${ }^{\mathrm{b}}$ Bushfire and Natural Hazards Cooperative Research Centre, Australia \\ ${ }^{\mathrm{c}}$ Climate Change Research Centre, University of New South Wales, Australia
}

Email: christopherthomas@cmt.id.au

\begin{abstract}
Ember transport and subsequent spot-fire development is an important mechanism of fire propagation, particularly in extreme conditions, and particularly in the eucalypt forests of Australia. Early modelling of ember transport used a combination of analytical and experimental work with appropriate simplifying assumptions. One of these simplifying assumptions is the so-called terminal-velocity assumption, in which embers are assumed to fall at all times at their terminal velocities with respect to the wind field. With the advent of high-speed computers, more elaborate sets of equations of motion can be solved numerically to model ember transport, and the terminal-velocity assumption has become less important. However, this adds computational cost and the assumption is sometimes still used.

Thurston et al. (2017) used the terminal-velocity assumption to study long-range ember transport in turbulent plumes using a large eddy simulation (LES) model. However, Koo et al. (2012) have modelled surface fire using a high-resolution coupled atmosphere-fire model and showed that simulations of short-range ember transport using the terminal velocity assumption underestimated ember travel distances substantially when compared with simulations in which the assumption was not made.

In this preliminary study the validity of the terminal-velocity assumption was examined in the context of the long-range transport of embers in turbulent plumes. The Weather Research and Forecasting (WRF) model (Skamarock et al., 2008) was used in LES mode to simulate a plume in a turbulent boundary layer. Using the resulting wind field, the transport of embers was modelled under various assumptions, and the results were compared. The effects of combustion were not considered. It was found that if a constant terminal velocity was assumed, then the use of the terminal-velocity assumption overestimated ember travel distances compared with simulations in which the assumption was not made. This effect was attributed partly to variations in atmospheric density, and partly to the fact that embers have momentum, and do not respond instantly to changes in the incident wind field.
\end{abstract}

Keywords: Ember transport, firebrands, spot fires, terminal-velocity assumption, turbulent plumes 


\section{INTRODUCTION}

Without access to high-speed computers, the early researchers modelling ember transport used a combination of experimental and analytical techniques with appropriate simplifying assumptions. Tarifa et al. (1965) wrote down the equations of motion of an ember moving at velocity $\mathbf{u}$ in a wind field $\mathbf{w}$ as

$$
\frac{\mathrm{d} \mathbf{u}}{\mathrm{d} t}=\frac{C_{\mathrm{d}} \rho A}{2 m}|\mathbf{v}| \mathbf{v}-g \mathbf{k}
$$

where $\mathbf{v}=\mathbf{w}-\mathbf{u}$ is the wind field relative to the ember, $C_{\mathrm{d}}$ is the ember's drag coefficient, $\rho$ is the atmospheric density, $m$ is the mass of the ember, $A$ it's projected area in the direction of $\mathbf{v}$, and $\mathbf{k}$ is the unit vector in the $z$-direction. In Eqn. (1) it is assumed that the aerodynamic force acts on the ember through its centre of mass, and in the direction of $\mathbf{v}$, i.e. any rotational motion of the ember is ignored, and the lift force is assumed to be zero. In general, each of the quantities in the term $\alpha \equiv C_{\mathrm{d}} \rho A / 2 m$ may vary as the ember moves within the atmosphere, loses mass due to drying and combustion, and changes orientation with respect to $\mathbf{v}$. Under the assumption that $\mathbf{w}$ and $\alpha$ are constant, Tarifa et al. (1965) obtained the explicit solution of Eqn. (1) and showed that, for typical values of $\alpha$, the solution rapidly approaches its asymptotic value of

$$
\mathbf{u}_{\infty}=\mathbf{w}-\sqrt{\frac{2 m g}{C_{\mathrm{d}} \rho A}} \mathbf{k}
$$

where $u_{\infty} \equiv \sqrt{2 m g / C_{\mathrm{d}} \rho A}$ is the terminal (fall) speed of the ember. They concluded that, for non-constant wind fields $\mathbf{w}$ varying over time scales much longer than a few seconds, there is little error in assuming that an ember always travels at its terminal velocity, $\mathbf{u}=\mathbf{u}_{\infty}$. This so-called terminal-velocity assumption was important in early studies of ember transport (e.g. Tarifa et al. (1965), Albini (1979)); it greatly simplifies analytical work, but has also been used when performing computer simulations (e.g. Koo et al. (2012), Thurston et al. (2017)).

Computer models, including coupled atmosphere-fire models, are now capable of high-resolution simulations of the atmospheric conditions associated with fires, and of explicitly resolving at least the larger turbulent processes in the wind field. Such models may be used to simulate ember transport. They are not restricted to making the terminal-velocity assumption, but can compute ember trajectories using Eqn. (1), or a more complete set of equations incorporating lift forces, rotational motion of the embers, and changes in ember characteristics due to combustion. Examples of studies using such models include Himoto and Tanaka (2005), Sardoy et al. (2007), Bhutia et al. (2010), Koo et al. (2012), and Pereira et al. (2015).

However, eschewing the terminal-velocity assumption adds an additional level of computational complexity, and the question arises as to whether the assumption can be justified when modelling ember transport in turbulent flows. Koo et al. (2012) simulated ember transport due to surface fires using the HIGRAD/FIRETEC coupled model (Linn et al., 2002), and found that simulated embers flew significantly further if the terminalvelocity assumption was not used; the travel distances they were simulating were of the order of $10^{0}$ to $10^{2}$ metres. In what appears to be the first numerical study of long-range ember transport, Thurston et al. (2017) studied the transport of embers in the plume produced by a static surface-heat-flux anomaly using a large eddy simulation (LES) model. They computed ember trajectories under the terminal-velocity assumption, setting $\mathbf{u}=\mathbf{u}_{\infty}$, with a constant terminal fall speed, $u_{\infty}$.

In the current work we make a preliminary investigation of the validity of the terminal-velocity assumption in the numerical modelling of long-range ember transport. We use the Weather Research and Forecasting (WRF) atmospheric model (Skamarock et al., 2008) in LES mode to simulate the plume from a static heat source. The resulting wind field is used to model ember transport under various assumptions, and the results are compared. Like Thurston et al. (2017), we do not model the combustion process; embers are treated as inert particles whose mass and geometry do not change during transport. There are various other simplications made; these are discussed in the model description below. Our aim is not to study spotting distances or develop distributions of ember fall; rather it is to work towards determining the level of model complexity that is appropriate for such studies.

\section{THE ModeL}

\subsection{Overview}

The modelling was performed in three stages: an atmospheric model was used to compute a turbulent boundary layer; a constant heat flux was then applied at the lower boundary to simulate a fire, and the atmospheric 
C. Thomas el al., Evaluating the terminal-velocity assumption...

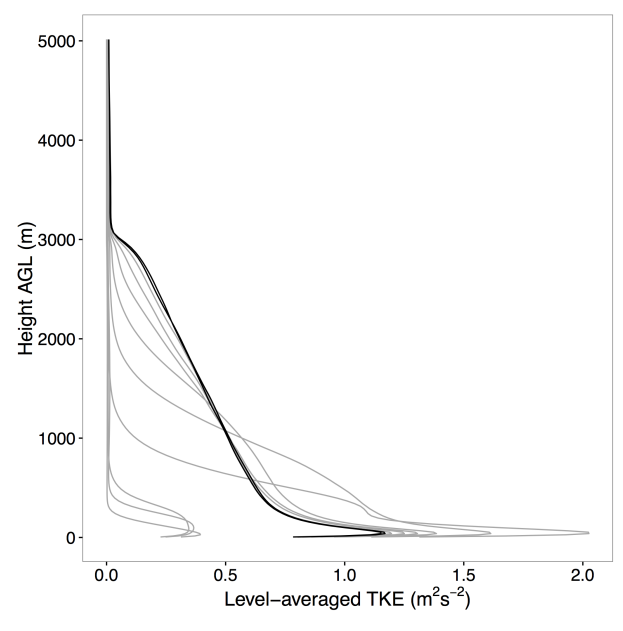

(a) Level-averaged TKE

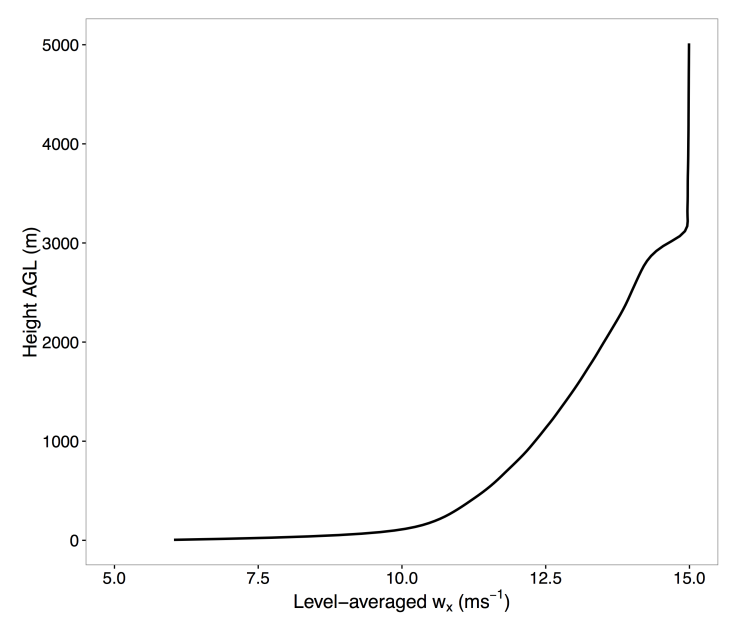

(b) Level-averaged $w_{x}$

Figure 1. (a) Evolution of total (subgrid scale + resolved) turbulence kinetic energy (TKE) during the spin-up phase of the simulation. The grey lines show the level-averaged TKE hourly for the first 9 hours. The black lines show the level-averaged TKE at $t=10: 00$ and $t=11: 00$ hours. (b) Level-averaged $x$-component of wind field, $w_{x}$, at $t=10: 00$ hours, immediately prior to application of sensible heat flux.

simulation was continued; finally, the resulting wind field was used as an input into simulations of ember transport. The first two stages of the modelling follow reasonably closely the procedure adopted by Thurston et al. (2013). This enables a comparison of their results using a different large eddy model. In the final stage we perform ember trajectory calculations, both with and without the terminal-velocity assumption.

\subsection{Turbulent boundary layer and simulated plume}

Simulations were performed using the WRF atmospheric model (Skamarock et al., 2008) in LES mode. The computational domain was $40 \times 16 \times 12$ kilometres $(x \times y \times z)$ with a horizontal resolution of $50 \mathrm{~m}$ and a stretched vertical grid of 256 levels. Initially, the vertical resolution varied smoothly from $\sim 10 \mathrm{~m}$ at the surface to $\sim 50 \mathrm{~m}$ at the model top; the precise vertical spatial resolution may fluctuate slightly during a simulation because of the pressure-based vertical coordinate system used by the WRF model (Skamarock et al., 2008). Periodic boundary conditions were used at the lateral boundaries, enabling recirculation of the developing turbulence as it is advected across the downwind boundary. The model was initialised with a 3-km-deep dryadiabatic layer at a constant potential temperature of $300 \mathrm{~K}$, topped by a stable layer with a lapse rate of $3 \mathrm{~K}$ per kilometre. To stimulate the development of turbulence, small random perturbations were introduced into the bottom $3 \mathrm{~km}$ of the initial temperature profile. The initial wind profile was set to a constant value of $w_{x}=15$ $\mathrm{ms}^{-1}$ over the depth of the model, with $w_{y}=w_{z}=0$. After 10 hours of model time, a turbulent boundary layer had developed; the vertical profile of turbulence kinetic energy (TKE) had stabilised (Figure 1(a)), and $w_{x}$ had assumed the vertical profile seen in Figure 1(b), consistent with a shear-driven turbulent boundary layer (Moeng and Sullivan, 1994). At this point in model time, the effects of a wildfire were simulated by introducing a constant surface sensible heat flux of $10^{5} \mathrm{Wm}^{-2}$ over a circular region of radius $250 \mathrm{~m}$, located $2 \mathrm{~km}$ inside the upwind boundary of the domain. The simulation was run for a further 30 minutes of model time to allow the ensuing plume to develop, and then for a further 60 minutes, during which simulation data were output at $5 \mathrm{~s}$ intervals. It is these high-temporal-resolution data which were used to simulate ember transport in the turbulent plume.

\subsection{Ember release and transport}

Preliminaries. Ember transport under the terminal-velocity assumption, as employed by Thurston et al. (2017) for example, is described by

$$
\mathbf{u}=\mathbf{w}-u_{\infty} \mathbf{k}
$$

where $u_{\infty}$ is a specified constant, the terminal speed of the ember. We will refer to this as the constantterminal-velocity assumption. In Eqn. (3), embers are assumed to have a constant terminal speed of $u_{\infty}$ regardless of their location whereas, according to Eqn. (2), their terminal speed depends on atmospheric density 
$\rho$. If we interpret $u_{\infty}$ as being the terminal speed of an ember at a reference atmospheric density $\rho_{0}$ then, from Eqn. (3),

$$
u_{\infty}=\sqrt{\frac{2 m g}{C_{\mathrm{d}} \rho_{0} A}} .
$$

In this study we set $\rho_{0}=1.16$, which is the approximate atmospheric density at sea level. This is a reasonable, but arbitrary, choice; a different choice of $\rho_{0}$ is simply equivalent to a scaling of the value of $u_{\infty}$.

Using Eqn. (4), we may rewrite Eqn. (1) as

$$
\frac{\mathrm{d} \mathbf{u}}{\mathrm{d} t}=g \frac{\rho}{\rho_{0}} \frac{1}{u_{\infty}^{2}}|\mathbf{v}| \mathbf{v}-g \mathbf{k} .
$$

The only characteristic of the ember explicit in Eqn. (5) is the constant $u_{\infty}$, its terminal speed at an atmospheric density of $\rho_{0}$. Note that $\rho$, the atmospheric density at the location of the ember, is not constant.

The differences between ember motion under Eqn. (3) and under Eqn. (5) are that under Eqn. (5) embers have momentum, and the density of the atmosphere is variable. The term $\rho / \rho_{0}$ appearing in Eqn. (5) can be significantly different from unity both at higher altitudes, and within the plume, and it is likely that this will be a factor contributing to any differences between ember motion under Eqn. (3) and Eqn. (5). An intermediate formulation is possible, which isolates this effect; the ember is assumed to always travel at its terminal velocity, which depends on the atmospheric density $\rho$ at the location of the ember. This is motion according to Eqn. (2) which, using Eqn. (4), we may rewrite as

$$
\mathbf{u}=\mathbf{w}-\sqrt{\frac{\rho_{0}}{\rho}} u_{\infty} \mathbf{k} .
$$

We will refer to this as the variable-terminal-velocity assumption.

Eqns. (3), (5), and (6) allow us to make a direct comparison of the results of ember transport under these various assumptions. In each case the only characteristic of the embers that is specified is $u_{\infty}$, the terminal speed at an atmospheric density of $\rho_{0}$. We need only specify a set of embers with prescribed initial positions and terminal velocities, and apply Eqns. (3), (5), and (6) to this set.

For a non-spherical ember, the quantities $C_{\mathrm{d}}$ and $A$ depend on the orientation of the ember with respect to the relative wind $\mathbf{v}$. Implicit in our formulation is the assumption that these remain constant during ember transport. This amounts to assuming that an ember always has the same orientation with respect to $\mathbf{v}$, or that the ember adopts this orientation over time scales much shorter than those over which $\mathbf{v}$ changes. This assumption is similar in nature to the terminal-velocity assumption. The investigation of its validity for long range ember transport is beyond the scope of this paper, however we note that it is supported to some extent by the studies of Ellis (2010) and Tarifa et al. (1967); in drop tests, embers either assumed an orientation of maximum drag, or tumbled but fell at a speed as if they had assumed such an orientation. Some numerical studies, including those of Koo et al. (2012) and Oliveira et al. (2014), include the rotational terms in the equations of motion.

Ember release. Embers were released every $5 \mathrm{~s}$ for the first 30 minutes of the 60 -minute period over which ember transport was studied. They were released $10 \mathrm{~m}$ apart in a three-dimensional grid pattern, at heights between 33 and $90 \mathrm{~m}$, in a region where the the updraft speed $w_{\mathrm{z}}$ exceeded $5 \mathrm{~ms}^{-1}$. This resulted in 3,989,475 embers being released over the 30-minute period.

Transport computation. In this study we used the standard second-order Runge-Kutta scheme with a time step of $0.05 \mathrm{~s}$ to integrate Eqn. (3), Eqn. (5), or Eqn. (6), storing the ember location every $5 \mathrm{~s}$. The simulated atmospheric data, which are available only at discrete locations and times, are interpolated linearly in time and space to obtain values of $\mathbf{w}(\mathbf{x}, t)$ and $\rho(\mathbf{x}, t)$. The initial conditions for Eqn. (3) or Eqn. (6) are simply the ember location $\mathbf{x}_{0}$ at ember release time $t_{0}$. The initial conditions for Eqn. (5) are taken to be $\mathbf{x}\left(t_{0}\right)=\mathbf{x}_{0}$ and $\mathbf{u}\left(t_{0}\right)=\mathbf{w}\left(\mathbf{x}_{0}, t_{0}\right)-u_{\infty} \mathbf{k}$. This ensures that in all cases the ember is initially travelling at approximately terminal velocity (neglecting the factor $\sqrt{\rho_{0} / \rho}$ ). The precise formulation of the initial conditions will have little effect on the model results. In this preliminary study we restrict our attention to values of $u_{\infty}=6 \mathrm{~ms}^{-1}$ and $8 \mathrm{~ms}^{-1}$, which are two of the values used by Thurston et al. (2017), and are consistent with experimental results of Ellis (2010). The same set of initial ember positions was used for both $6 \mathrm{~ms}^{-1}$ and $8 \mathrm{~ms}^{-1}$ fall speeds. Computation times for transport calculations under Eqn. (5) were only slightly longer than those for Eqn. (3). However, the difference may be greater if a higher-order computational scheme (for example, a fourth-order Runge-Kutta scheme) was used. 
C. Thomas el al., Evaluating the terminal-velocity assumption...

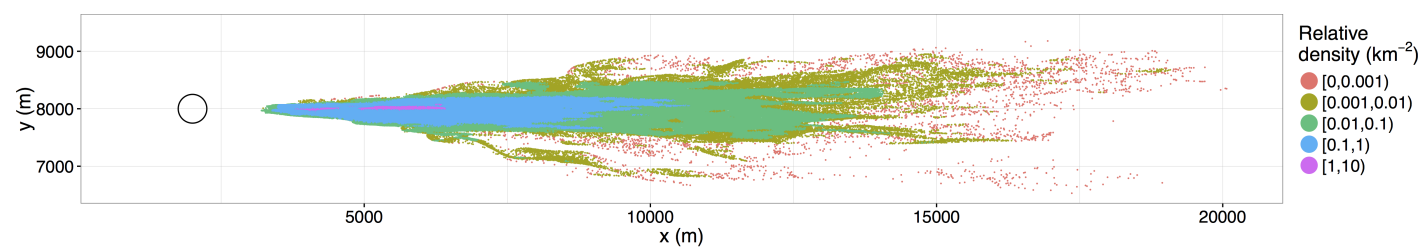

(a) Constant-terminal-velocity assumption

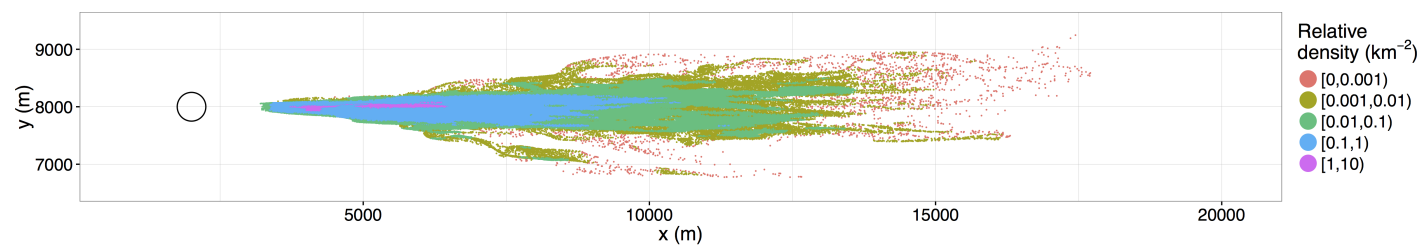

(b) Variable-terminal-velocity assumption

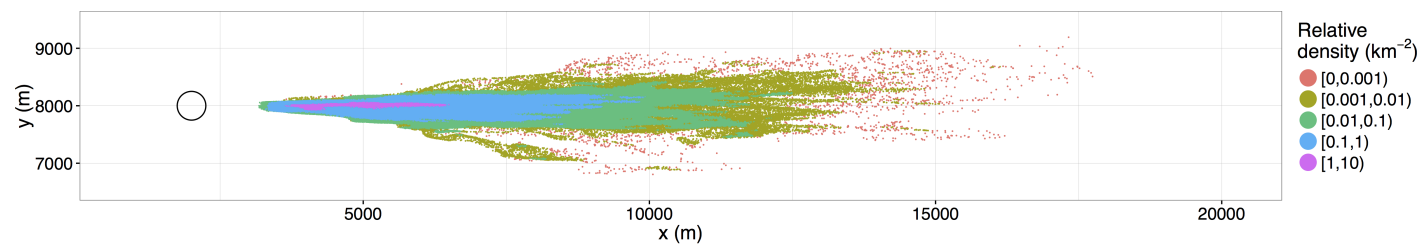

(c) No terminal-velocity assumption

Figure 2. Location and density of ember landings for embers with $u_{\infty}=6 \mathrm{~ms}^{-1}$ under: (a) Eqn. (3), the constant-terminal-velocity assumption; (b) Eqn. (6), the variable-terminal-velocity assumption; (c) Eqn. (5), no terminal-velocity assumption. Only embers that were transported at least $1 \mathrm{~km}$ under all three assumptions are included, 993,757 in total. Colours represent the density of landings per $\mathrm{km}^{2}$ as a proportion of the total landings, computed using a Gaussian kernel density estimator. The circle shows the location and extent of the sensible heat flux anomaly used to generate the plume.

\section{RESULTS AND DISCUSSION}

Figure 2 shows the two-dimensional landing distributions under the three transport assumptions for embers with $u_{\infty}=6 \mathrm{~ms}^{-1}$. The points are coloured according to the density of landings at each location, estimated using a Gaussian kernel density estimator. Travel distances under the constant-terminal-velocity assumption are clearly greater than those under no terminal velocity assumption, with the variable terminal velocity assumption assuming an intermediate position. Similarly, Figure 3 shows the landing distributions for embers with $u_{\infty}=8 \mathrm{~ms}^{-1}$; travel distances are greatest under the constant-terminal-velocity assumption, although in this case the landing distribution under the variable-terminal-velocity assumption appears quite similar to that under the constant-terminal-velocity assumption. We attribute this to the fact that, compared to the case of $u_{\infty}=6 \mathrm{~ms}^{-1}$, when $u_{\infty}=8 \mathrm{~ms}^{-1}$ the embers do not fly as high, and spend less time in the plume. This means that the factor $\rho / \rho_{0}$ in Eqn. (6) is closer to unity for more of the time that the ember is flying. In all cases it appears that the two-dimensional landing distributions are similar for medium to short range ember transport, i.e. for those embers that fly less than, say, 7,500 $\mathrm{m}$. This is borne out by Figure 4, which shows the distribution of travel distances. It is in the tail of the distribution that the greatest differences appear between ember travel distances under the constant-terminal-velocity assumption and under no terminal velocity assumption. Furthermore, it is the tail of the distribution which is most important in the modelling of long-range spot-fire development.

In their study, Koo et al. (2012) found that using the terminal-velocity assumption led to an underestimate of ember travel distances, attributing this to embers being 'thrown by locally strong winds' when modelled without the terminal-velocity assumption. While our results may appear at odds with this, it must be remembered that their simulations were of smaller-scale phenomena; they used a domain with horizontal dimensions $640 \times$ $320 \mathrm{~m}$, and they reported maximum travel distances of the order of $10^{1}$ to $10^{2} \mathrm{~m}$. Maximum travel distances in our simulations exceed $20 \mathrm{~km}$, and we are modelling embers which remain in the turbulent plume for extended periods. The reasons underlying our results will be investigated as part of a future study.

The modelling and analysis presented here is greatly simplified. For one thing, we are not considering com- 
C. Thomas el al., Evaluating the terminal-velocity assumption...

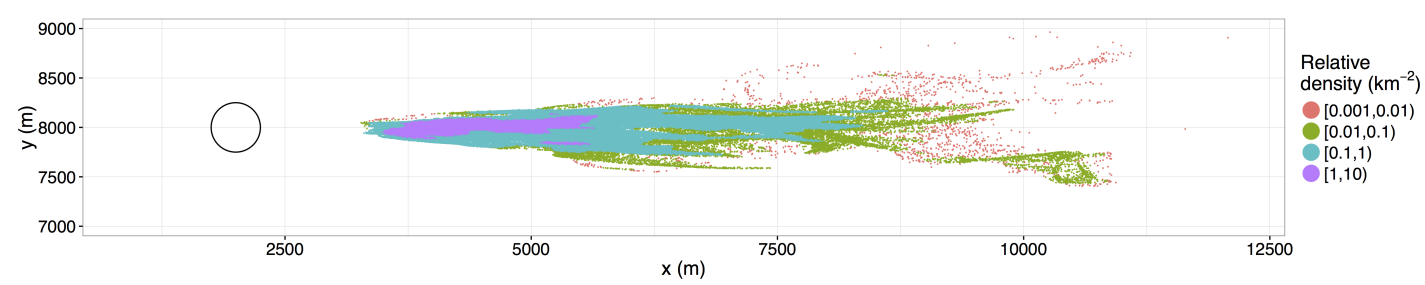

(a) Constant-terminal-velocity assumption

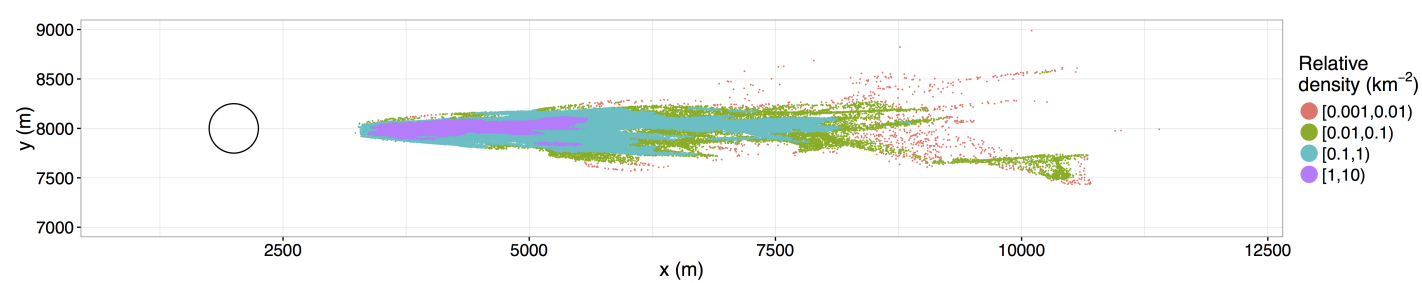

(b) Variable-terminal-velocity assumption

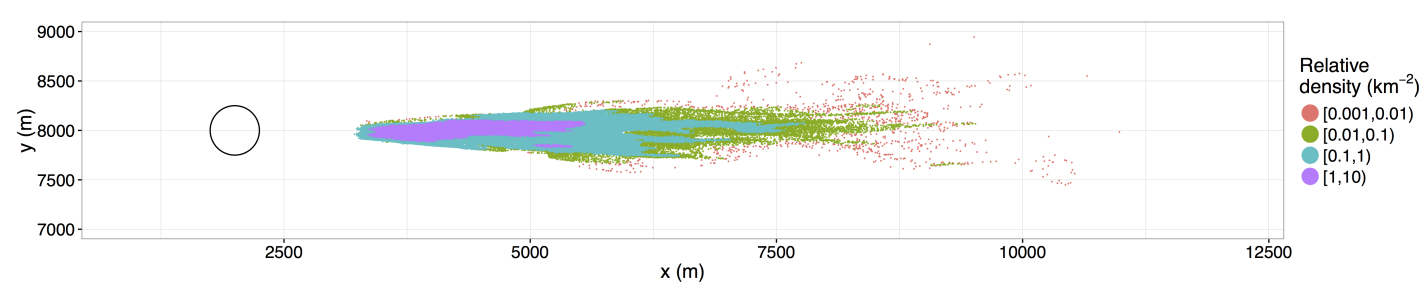

(c) No terminal-velocity assumption

Figure 3. As for Figure 2 but for embers with $u_{\infty}=8 \mathrm{~ms}^{-1}$.

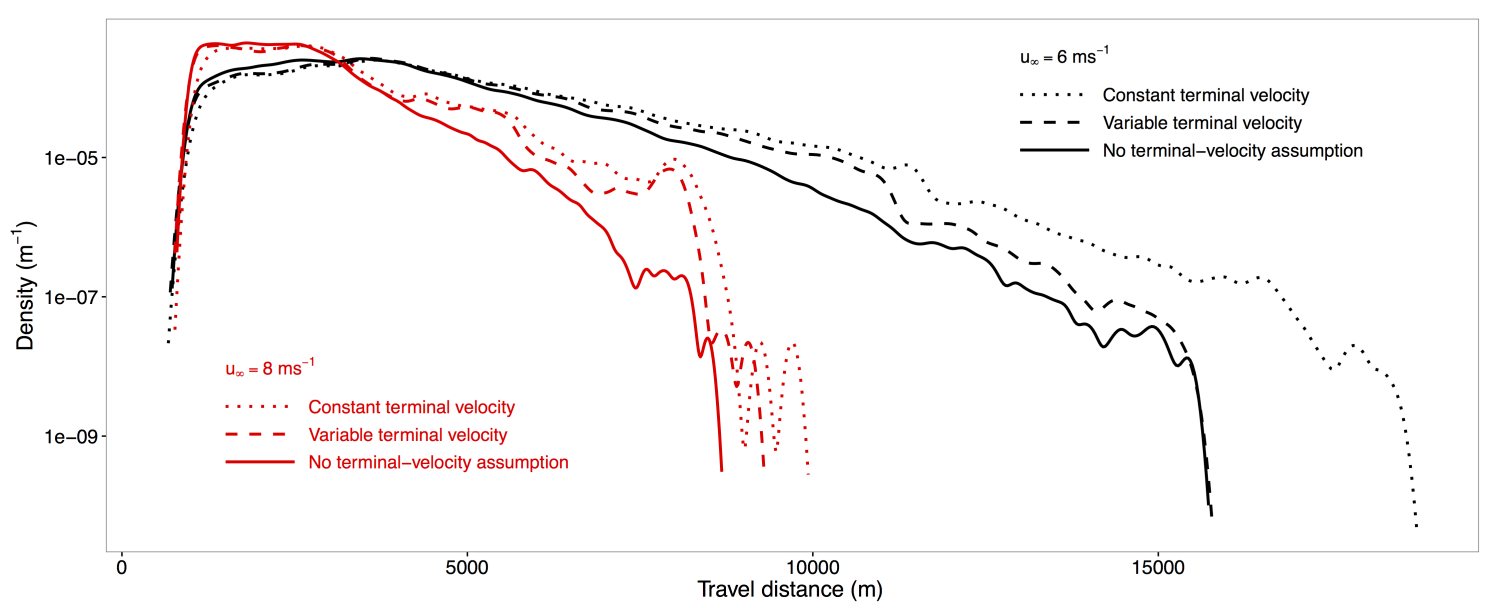

Figure 4. Distribution of travel distances for embers that have travelled further than $1 \mathrm{~km}$, computed using a Gaussian kernel density estimator.

bustion, which affects many aspects of ember transport including the value of $\rho$ in Eqn. (1) and Eqn. (2). However, the results show that it is not just the variable nature of $\rho$ that leads to the differences in modelled trajectories under Eqn. (4) and Eqn. (5). In any case, our aim here is not to produce meaningful ember-landing distributions, but to examine whether the terminal-velocity-assumption is justified in studies of long-range ember transport. Our results indicate that it is not.

\section{Conclusion}

Under simplifying assumptions we have used a LES model to make a comparison of three methods of computing ember trajectories in a numerical study of long-range ember transport. The only characteristics of an 
C. Thomas el al., Evaluating the terminal-velocity assumption...

ember that we have used are its initial position, and its terminal fall speed at a given reference atmospheric density. We have not considered the effects of combustion in our analysis. We have found that the use of the terminal velocity assumption overestimates ember travel distances when compared with calculations in which this assumption is not made, and that this difference is, statistically, greater for those embers that travel further.

\section{ACKNOWLEDGEMENT}

This research has been supported by The Bushfire and Natural Hazards Cooperative Research Centre and was undertaken with the assistance of resources provided at the NCI National Facility systems at the Australian National University through the National Computational Merit Allocation Scheme supported by the Australian Government.

\section{REFERENCES}

Albini, F. A. (1979, July). Spot fire distance from burning trees - a predictive model. Technical report, Intermountain Forest and Range Experiment Station, Ogden, Utah 84401, Ogden, Utah.

Bhutia, S., M. Ann Jenkins, and R. Sun (2010). Comparison of Firebrand Propagation Prediction by a Plume Model and a CoupledFire/Atmosphere LargeEddy Simulator. Journal of Advances in Modeling Earth Systems 2(1), 1-15.

Ellis, P. F. M. (2010). The effect of the aerodynamic behaviour of flakes of jarrah and karri bark on their potential as firebrands. Journal of the Royal Society of Western Australia 93, 21-27.

Himoto, K. and T. Tanaka (2005). Transport of Disk-shaped Firebrands in a Turbulent Boundary Layer. In D. T. Gottuk and B. Y. Latimer (Eds.), Proceedings of the Eighth International Symposium on Fire Safety Science. International Association for Fire Safety Science.

Koo, E., R. R. Linn, P. J. Pagni, and C. B. Edminster (2012). Modelling firebrand transport in wildfires using HIGRAD/FIRETEC. International Journal of Wildland Fire 21(4), 396-417.

Linn, R., J. Reisner, J. J. Colman, and J. Winterkamp (2002). Studying wildfire behavior using FIRETEC. International Journal of Wildland Fire 11(4), 233-246.

Moeng, C.-H. and P. P. Sullivan (1994, April). A Comparison of Shear- and Buoyancy-Driven Planetary Boundary Layer Flows. Journal of the Atmospheric Sciences 51(7), 999-1022.

Oliveira, L. A., A. G. Lopes, B. R. Baliga, M. Almeida, and D. X. Viegas (2014, August). Numerical prediction of size, mass, temperature and trajectory of cylindrical wind-driven firebrands. International Journal of Wildland Fire 23(5), 698-708.

Pereira, J., C. F, J. Pereira, M. C, A. Leite, L. A, and D. M. S. Albuquerque (2015). Calculation of Spotting Particles Maximum Distance in Idealised Forest Fire Scenarios. DOI: 10.1155/2015/513576.

Sardoy, N., J.-L. Consalvi, B. Porterie, and A. C. Fernandez-Pello (2007, August). Modeling transport and combustion of firebrands from burning trees. Combustion and Flame 150(3), 151-169.

Skamarock, W. C., J. B. Klemp, J. Dudhia, D. O. Gill, D. M. Barker, M. Duda, X. Y. Huang, W. Wang, and J. G. Powers (2008). A description of the advanced research WRF version 3. NCAR Technical Note NCAR/TN475+STR, National Centre for Atmospheric Research, Boulder, Colorado, USA.

Tarifa, C. S., P. P. del Notario, and F. G. Moreno (1965). On the flight paths and lifetimes of burning particles of wood. In Tenth Symposium (International) on Combustion, pp. 1021-1037.

Tarifa, C. S., P. P. del Notario, F. G. Moreno, and A. R. Villa (1967). Transport and combustion of firebrands. Technical report, Instituto Nacional de Tecnica Aeronautica, Madrid.

Thurston, W., J. D. Kepert, K. J. Tory, and R. J. B. Fawcett (2017, May). The contribution of turbulent plume dynamics to long-range spotting. International Journal of Wildland Fire 26(4), 317-330.

Thurston, W., K. J. Tory, R. J. B. Fawcett, and J. D. Kepert (2013). Large-eddy simulations of bushfire plumes in the turbulent atmospheric boundary layer. In MODSIM2015, 21st international congress on modelling and simulation, pp. 284-289. 